\title{
miR2118-triggered phased siRNAs are differentially expressed during the panicle development of wild and domesticated African rice species
}

\author{
K. N. Ta ${ }^{1,2+}$, F. Sabot ${ }^{1 \dagger}$, H. Adam ${ }^{1}$, Y. Vigouroux ${ }^{1}$, S. De Mita ${ }^{1,4}$, A. Ghesquière ${ }^{1}$, N. V. Do ${ }^{2}$, P. Gantet ${ }^{2,3}$
} and S. Jouannic ${ }^{1,2^{*}}$

\begin{abstract}
Background: Rice exhibits a wide range of panicle structures. To explain these variations, much emphasis has been placed on changes in transcriptional regulation, but no large-scale study has yet reported on changes in small RNA regulation in the various rice species. To evaluate this aspect, we performed deep sequencing and expression profiling of small RNAs from two closely related species with contrasting panicle development: the cultivated African rice Oryza glaberrima and its wild relative Oryza barthii.

Results: Our RNA-seq analysis revealed a dramatic difference between the two species in the 21 nucleotide small RNA population, corresponding mainly to miR2118-triggered phased siRNAs. A detailed expression profiling during the panicle development of O. glaberrima and O. barthii using qRT-PCRs and in situ hybridization, confirmed a delayed expression of the phased siRNAs as well as their InCRNA precursors and regulators (miR2118 and MEL1 gene) in 0 . glaberrima compared to 0 . barthii. We provide evidence that the 21-nt phasiRNA pathway in rice is associated with male-gametogenesis but is initiated in spikelet meristems.
\end{abstract}

Conclusion: Differential expression of the miR2118-triggered 21-nt phasiRNA pathway between the two African rice species reflects differential rates of determinate fate acquisition of panicle meristems between the two species.

Keywords: Panicle, Meristem, Small RNAs, phasiRNAs, miR2118, Domestication, Oryza barthii, Oryza glaberrima

\section{Background}

A prevailing view in evolutionary developmental biology (Evo-Devo) is that morphological traits evolved mostly by changes in expression patterns of functionally conserved genes rather than through the emergence of new genes (Doebley and Lukens 1998; Carroll 2008). Although much emphasis was placed on changes in transcriptional regulation, gene expression is regulated at many levels. In this context, the regulation of genome expression by small RNAs appears to be an important

\footnotetext{
* Correspondence: stephane.jouannic@ird.fr

${ }^{\dagger}$ Equal contributors

${ }^{1}$ IRD, UMR DIADE, 911, avenue Agropolis, BP64501, F-34394 Montpellier,

Cedex 5, France

${ }^{2}$ LMI RICE, National Key Laboratory for Plant Cell Biotechnology, Agronomical Genetics Institute, Pham Van Dong road, Hanoi, Vietnam

Full list of author information is available at the end of the article
}

mechanism in the control of plant development (morphogenesis and phase transition), through posttranscriptional regulation of mRNA abundance (by $m i R$ $N A s$ and secondary siRNAs) and silencing of gene expression (by siRNAs) via site-specific DNA methylation (Jones-Rhoades et al. 2006; Arikit et al. 2013). Secondary, phased small interfering RNAs (phasiRNAs) are emerging regulators of gene expression in plants (Zheng et al. 2015; Xia et al. 2013). Analyses in large panel of plant species covering plant kingdom revealed that a huge diversity of phasiRNA-generating loci types in plants (Zheng et al. 2015; Xia et al. 2013). An unsuspected diversity of phasiRNAs originating from proteincoding genes was observed. However, most of these loci were clade- or species-specific with distinct expression 
patterns, suggesting recent and independent evolutionary origins (Xia et al. 2013).

Several types of phasiRNA-generating loci were reported from Asian rice Oryza sativa and its wild relative Oryza rufipogon from various tissues or organs (Arikit et al. 2013; Zheng et al. 2015; Liu et al. 2013). In O. sativa, phasiRNAs triggered by specific miRNAs and originating from non-coding loci, were shown to be involved in panicle development and more specifically in male gametogenesis (Arikit et al. 2013; Johnson et al. 2009; Komiya et al. 2014; Song et al. 2012a, b). The TAS3-associated trans-acting siRNAs (or ta-siRNAs), triggered by the microRNA miR390, are known to target mRNAs of Auxin Response Factor (ARFs) involved in various developmental processes including floret and stamen development (Allen et al. 2005; Liu et al. 2007; Nogueira et al. 2007; Song et al. 2012b). Other detected phasiRNAs are panicle-specific 21- or 24-nucleotide (nt) small RNAs. They are produced from numerous polyA-tailed long non-coding RNA (lncRNA) generating loci through an RDR6-dependent pathway triggered by the microRNAs miR2118 and miR2275 respectively (Komiya et al. 2014; Song et al. 2012a, b). The 21-nt phasiRNAs may act in a complex with the gamete-specific Argonaute (AGO) protein MEIOSIS ARRESTED AT LEPTOTENE1 (MEL1) (Komiya et al. 2014). In maize, each phasiRNA type exhibits independent spatiotemporal regulation, with 21-nt premeiotic phasiRNAs dependent on stamen epidermal differentiation and 24-nt meiotic phasiRNAs dependent on tapetal cell differentiation (Zhai et al. 2015). However, their function during male gametogenesis remains unclear.

African rice Oryza glaberrima was domesticated about 2000 to 3000 years ago from Oryza barthii, whereas $O$. sativa was domesticated in Asia almost 10,000 years ago from Oryza rufipogon (Second 1982; Linares 2002; Caicedo et al. 2007; Vaughan et al. 2008; Huang et al. 2012; Orjuela et al. 2014; Wang et al. 2014; Zhang et al. 2014a. African rice domestication has a single origin in West Africa (Linares, 2002; Wang et al. 2014) and is associated with a severe genetic bottleneck ( $\mathrm{Li}$ et al. 2011a, b; Nabholz et al. 2014; Orjuela et al. 2014). It is also associated with major morphological and physiological changes. For example, O. barthii and O. glaberrima exhibit strikingly different panicle architectures, from a low branching complexity and small grain number in $O$. barthii to a more complex panicle and higher grain number in O. glaberrima (Linares 2002). A similar divergence of domestication-related traits was also observed between the Asian wild rice and its cultivated counterpart (Vaughan et al. 2008). It has been suggested that several small RNA loci, such as miRNA-triggered phasiRNAs loci including ta-siRNA locus TAS3a2, as well as the microRNA loci MIR164e, MIR390 and
MIR395a/b, have experienced direct selection during Asian rice domestication (Liu et al. 2013; Wang et al. 2010; Wang et al. 2012). However, a large-scale study of small RNA expression in relation to the early stages of panicle development in African rice is still lacking. To investigate whether the latter varies between the two African species in relation to changes in small RNA expression, we conducted a comparative analysis of small RNA populations accumulating during early panicle development in the two African species. We found conservation of the male gametogenesis-associated miR2118-triggered phasiRNA pathway in these African rice species. Our data revealed significant changes in expression of these gamete-specific 21-nt phasiRNAs between $O$. barthii and O. glaberrima, associated with differential expression of their regulators, namely miR2118 and MEL1. This is suggestive of a differential rate of determinate meristem fate acquisition during the initial panicle development stages between the two species.

\section{Results}

\section{Panicle-associated small RNAs in African rice species}

To conduct a comprehensive survey of panicle-derived small RNAs in African rice and characterise qualitative and quantitative differences between the two species, we compared small RNA populations in panicles of O. glaberrima and $O$. barthii, using genome-wide small RNA-seq analysis. In order to focus on differences in expression resulting from inter-specific variations and to buffer genotypic variations, we used two RNA bulks extracted from developing panicles at same visual stage (from early rachis elongation to early floret differentiation stages) of 10 genotypes for each species (Additional file 1: Table S1). Over 33.1 and 33.9 million high quality reads were generated from $O$. glaberrima and $O$. barthii libraries respectively (Additional file 1: Table S2). A total of $64 \%$ and $64.5 \%$ of small RNA clusters (distinct small RNAs) ranging from 18 to 28 nucleotides from $O$. barthii and O. glaberrima, respectively, were mapped to the reference genome $O$. sativa ssp japonica cv Nipponbare MSU v7.0, and were found to be similarly distributed over the $O$. sativa genome (Additional file 1: Table S3, Additional files 2 and 3). As expected, the 21- and 24-nucleotide (nt) small RNAs were the predominant populations of small RNAs in the two species, with a higher number of distinct 24-nt small RNAs (Additional file 2). The high quality small RNA sequences were then categorized, using a BLAST-based filtering annotation pipeline (Additional file 4), into five distinct classes corresponding to the different functional compartments of the rice genome: miRNAs, ncRNAs, repeats, genes (CDS, intron, UTR) and unannotated regions of $O$. sativa reference genome (Additional file 1: Table S4).

The 21-nt small RNA population from the two species, corresponding to 23798 distinct sequences, exhibited a 
surprisingly large fraction of small RNAs with a higher expression in O. barthii than in O. glaberrima (Fig. 1a). This subpopulation corresponds to $29 \%$ of the $21-n t$ small RNAs mapped, when considering small RNAs that were at least 5-fold more accumulated in $O$. barthii. Other size classes (from 18- to 28-nt, except 21-nt) were not distinguished by such a pattern of distribution between the two species (Additional file 5). The overall 21-nt small RNAs were categorized into five distinct classes corresponding to the different functional compartments of the rice genome: miRNAs (11.3\%), ncRNAs (13.1\%), repeats (12.4\%), genes (CDS, intron, UTR: $25.5 \%$ ) and unannotated regions
(37.7 \%) of O. sativa reference genome. However, the distribution of the over-expressed fraction in the wild species showed a strong bias towards unannotated regions $(81.4 \% ; p$-value $=0.0)$ with the remaining classes all severely under-represented: miRNAs $(2.5 \%$; $p$-value $\left.=8.0 \times 10^{-139}\right)$, ncRNAs $(0.6 \% ; p$-value $=3.5 \times$ $\left.10^{-301}\right)$, repeats $\left(0.8 \% ; p\right.$-value $\left.=2.3 \times 10^{-261}\right)$ and genes $\quad\left(14.8 \%\right.$; $p$-value $\left.=1.2 \times 10^{-83}\right) \quad($ Fig. $\quad 1$ a; Additional file 5). This indicated that the overexpressed 21-nt small RNAs detected in O. barthii panicles were mainly derived from unannotated regions of the $O$. sativa reference genome (Fig. 1a; Additional file 5).

\section{(A)}

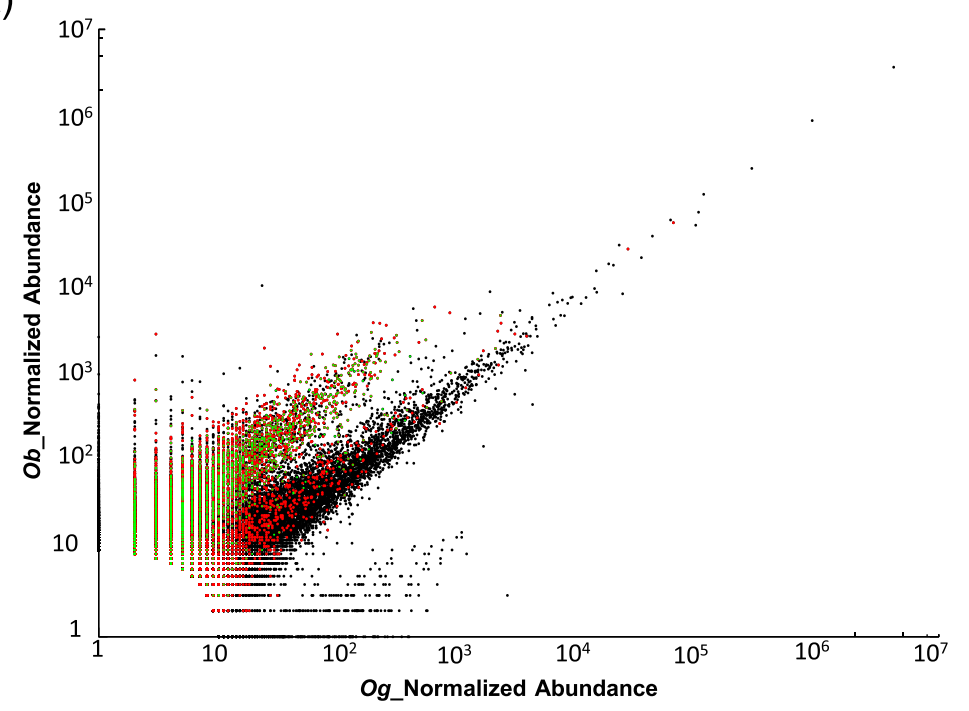

(B)

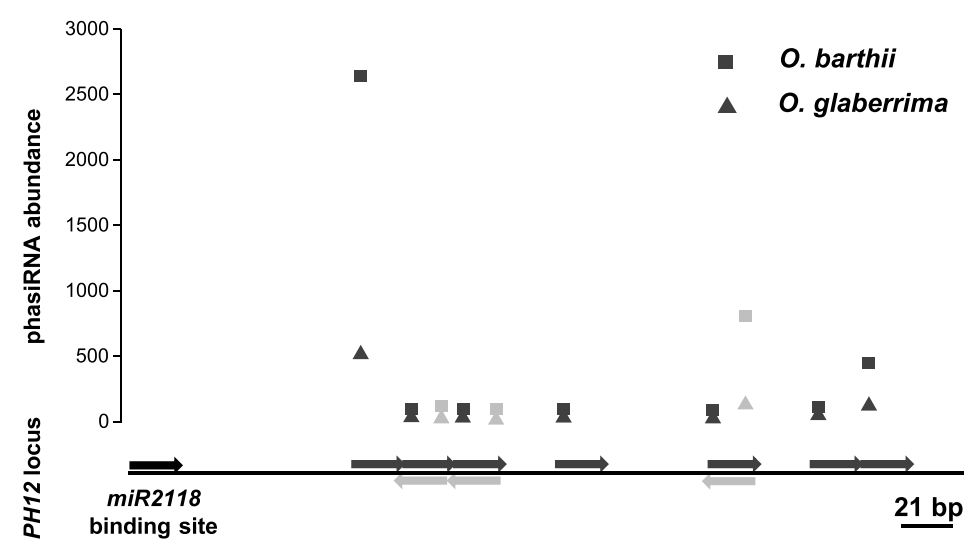

Fig. 1 Relative abundance of panicle-derived 21-nt small RNAs from O. barthii and O. glaberrima. a Relative abundance of 21-nt small RNAs between $O$. barthii $(\mathrm{Ob})$ and $\mathrm{O}$. glaberrima $(\mathrm{Og})$. Normalized abundance of distinct small RNA sequences. Black dots represent global 21-nt small RNAs, red dots unannotated 21-nt small RNAs, and green dots 21-nt small RNAs associated with detected phased loci. b Relative abundance of detected phasiRNAs generated from a single locus (PH-12) between O. barthii and O. glaberrima. The lower panel illustrates the position of the miR2118 binding site and the detected phasiRNAs from the PH12 locus in the two species (above or below the line if originating from plus or minus strand respectively). The upper panel illustrates the abundance of the corresponding phasiRNAs in $O$. glaberrima (triangles) and $O$. barthii (squares). The abundances of reads from the plus strand are in dark grey, and reads from the minus strand are in light grey 
21-nt phasiRNAs are over-represented in African wild rice transcriptomes

Previous studies of $O$. sativa showed that the miR2118triggered 21-nt phasiRNAs specifically expressed in the rice panicle originate from unannotated regions of the genome (Johnson et al. 2009; Song et al. 2012a; Komiya et al. 2014). To determine whether the over-represented 21-nt small RNA fraction in $O$. barthii also corresponds to this class of 21-nt phasiRNAs, we used a dedicated program (see Material and methods), and detected 4100 distinct phasiRNAs from $O$. barthii and O. glaberrima, distributed across 892 loci (denoted "phased loci"), using the $O$. sativa genome as reference (Additional file 6). In addition, 952 distinct 21-nt small RNAs mapped to these 892 loci, but were not detected as phased siRNAs by our procedure. In total, 5052 distinct 21-nt small RNA sequences associated with these 892 phased loci were found, corresponding to $21.2 \%$ of the panicle-derived 21-nt small RNA population from the two species. Out of these 5052 small RNAs, 3 694 were detected in both species, 1352 were detected only in O. barthii and six only in O. glaberrima (Additional file 6). As previously reported, the detected phased loci were present on all 12 chromosomes but not at the same density, and were organized in clusters or super-clusters, such as on chromosome 12 (Additional file 7). As expected, $86.5 \%$ of these 21-nt phasiRNAs mapped to unannotated regions of the O. sativa genome. This fraction of 21-nt phasiRNAs represents $49 \%$ of the unannotated 21-nt small RNA population (4 418 over 8977 sequences). The 892 phased loci were scanned for the presence of the 22-nt miRNA miR2118 recognition site in their vicinity (Johnson et al. 2009; Song et al. 2012a; Komiya et al. 2014). 529 phased loci $(59.3 \%)$ shared the conserved motif close to one end (from two to $452 \mathrm{bp}$ from the end). Between two and 91 distinct sequences were detected per phased locus, with an average of $6.0( \pm 4.8, \mathrm{SD})$ distinct sequences, the majority being 3 or 5 sequences per phased locus (Additional file 5). As expected for double strand RNA processed small RNAs, most of the detected phased loci generated small RNAs from both strands (92.5\%) (Additional file 6).

For $96 \%$ of the phased loci, there was a significant difference in read counts between the two species, and about $71 \%$ of them displayed an abundance ratio higher than 5 (Additional file 6). These 21-nt siRNAs were mainly over-accumulated in $O$. barthii and contributed to $52 \%$ of the $O$. barthii five fold over-accumulated 21 nt small RNAs (Fig. 1a). The abundance of different phasiRNAs from a given locus was variable, with the predominance of one or two phasiRNAs (Fig. 1b; Additional file 8). For the differentially expressed loci, this unequal distribution over the locus was conserved between the two species, with over-accumulation of all the detected phasiRNAs from a single locus (Fig. 1b). Interestingly, the proportion of phased loci sharing the miR2118 recognition site was significantly higher ( $p$ value $=1.5 \times 10^{-08}$ ) in the differentially expressed group (i.e. higher expression in $O$. barthii) $(61 \%, n=854)$ than in the group of non-differentially expressed phased loci (16\%, $n=38$ ) (Additional file 6). Therefore, phased loci over-expression in $O$. barthii versus $O$. glaberrima may result from differences in miR2118 regulation between the two species.

\section{Panicle-associated microRNAs in African rice species}

Comprehensive analysis of conserved miRNAs led to the identification of 146 annotated miRNA families expressed in African rice panicles, including 62 canonical families, 20 variant ones and 64 siRNA-like ones, according to the classification of Jeong et al. (2011) (Additional files 6 and 9). Some of the siRNA-like families were previously reported to be associated with transposable elements (TEs) in O. sativa, namely TE-MIRs ( $\mathrm{Li}$ et al. 2011b; Yan et al. 2011). In the same way, other annotated MIR loci were evidenced in our analysis of 21-nt phased loci as overlapping loci, suggesting that these loci are phasiRNA-generating loci rather than MIR ones: osa-MIR5486, osa-MIR5488, osa-MIR5506, osa-MIR5514, osa-miR5516, osa-miR5519, osa-MIR5517, osa-MIR5527, osa-MIR5530, osa-MIR5791, osa-MIR5796, osa-MIR5800 and osa-MIR5822 (Additional file 6). Thus, the annotations of these two classes of $M I R$ loci (i.e. variant and siRNA-like) should be reconsidered. Similarly to what was reported for $O$. sativa (Jeong et al. 2011), the canonical and variant miRNA families expressed in African rice panicles can be classified according to the length of the highest accumulated small RNA: length ranging from 19- to 25-nt, with 3 main types corresponding to 21-nt (48\%), 24-nt (31 \%) and 22-nt (9\%) long mature miRNAs (Additional files 6 and 9).

Most miRNA families were expressed at similar levels in $O$. barthii and O. glaberrima. Interestingly, the miR159/ 319 families contributed to $64 \%$ of mature miRNA expressed in young panicles of both African species (Additional file 6), in contrast to previous studies of panicle-derived miRNAs in O. sativa (Jeong et al. 2011; Peng et al. 2011). This may be related to the specific developmental stages used in our study or may reflect differences between Asian and African rice species. Differential expression affected only a few miRNA families with a trend towards higher expression in O. barthii (Fig. 2). These families included the canonical 22-nt miR2118 and five other ones, namely miR2275, miR5495, miR5497, miR5516 and miR5519 (Fig. 2). The latter two were identified in our study as panicle-expressed 21-nt phasiRNAs (Fig. 2; Additional file 6). The canonical 22-nt miRNA miR2275 was previously reported to trigger 24-nt phasiRNAs (Johnson et al. 2009; Song et al. 2012a). However, 


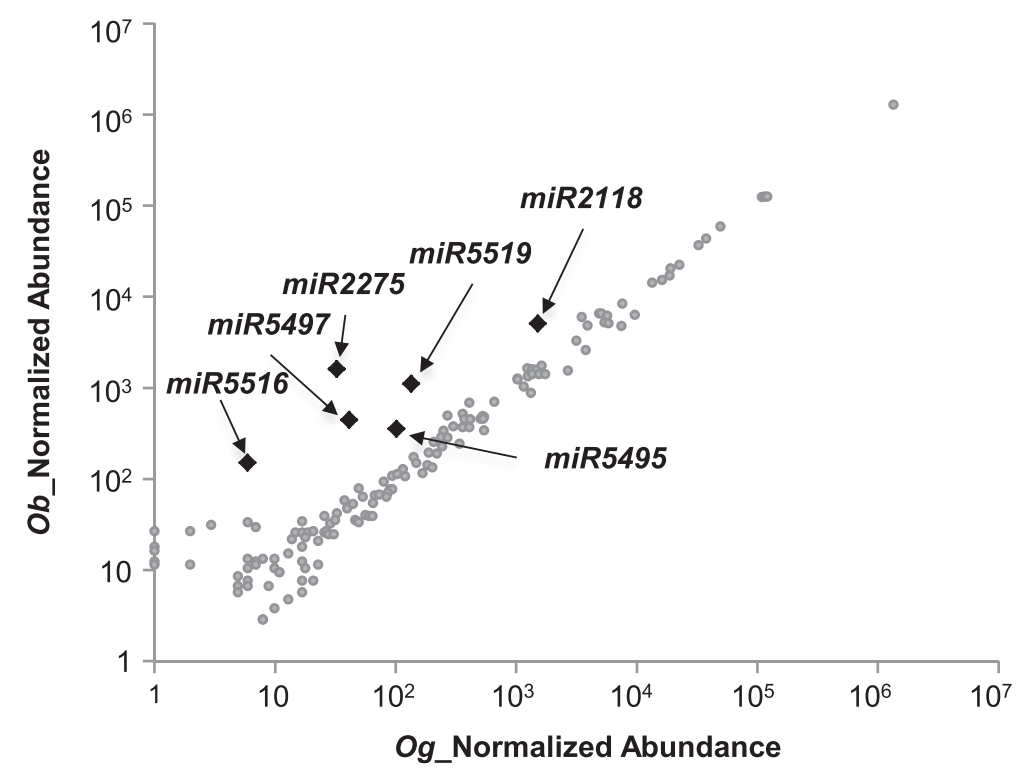

Fig. 2 Relative abundance of miRNA families between $O$. barthii $(\mathrm{Ob})$ and $\mathrm{O}$. glaberrima $(\mathrm{Og})$. Normalized abundance of miRNA reads. Black diamonds represent the differentially expressed miRNA families, and grey points to non-differentially expressed miRNA families between $O$. barthii and O. glaberrima

despite over-expression of miR2275 in O. barthii, no major over-expression of a large subset of the 24-nt small RNAs was detected (Additional file 5). The canonical miRNAs miR5495 and miR5497 were previously shown in $O$. sativa to be miRNAs specifically expressed in pollen (Wei et al. 2011).

Variations in the expression of miR2118, 21-nt phasiRNAs and associated precursors during African rice panicle development

The differential accumulation of specific microRNAs and 21-nt phasiRNAs observed between $O$. barthii and O. glaberrima may result from differences in their expression levels, but may also reflect a shift in the timing of their expression during panicle development. To test these hypotheses, expression analysis of miR2118, 21-nt phasiRNAs and their RNA precursors was performed on 4 distinct morphological stages of panicles collected from O. barthii (accession B88) and O. glaberrima (variety CG14) (Fig. 3; see Additional file 10 for histological description). Four different phased loci from distinct clusters and chromosomes were considered for the RNA precursors as well as the phasiRNA with the highest level of accumulation for each locus (Fig. 3; Additional files 8, 11 and 12).

The differential accumulation of miR2118, selected phasiRNAs and their corresponding precursors between the two species were validated through Northernblotting and/or RT-PCRs on RNA bulks similar to the ones used for small RNA deep sequencing (Additional file 8). While neither mature miR2118 nor 21-nt
phasiRNA phasiPH12-1 were detected at the early morphological stage of panicle development (inflorescence meristem after reproductive transition, stage 1), their expression was initiated earlier in $O$. barthii (early branching stage, stage 2) than in O. glaberrima (late branching stage, stage 3 ). The expression level reached a peak at stage 3 in $O$. barthii and remained at the same level at stage 4 (young flowers with differentiated organs). In $O$. glaberrima the miRNA and phasiRNA were more abundant at stage 4 and displayed a higher expression level than in $O$. barthii at this stage (Fig. 3). Similar patterns of expression were observed during panicle development for 21-nt phasiRNAs from the four phased loci tested (Additional file 12). These findings indicate that the later accumulation of 21-nt phasiRNAs during the panicle developmental time-course in O. glaberrima is associated with a later accumulation of miR2118. These findings are in agreement with the small RNA-seq data, as the RNA bulks used for sequencing originate from panicles at morphological stages 1 to 3 , in which $m i R 2118$ and 21-nt phasiRNAs are accumulated at higher levels in $O$. barthii than in O. glaberrima.

Similarly to Komiya et al. (2014), we showed that the 21-nt phasiRNAs from the African species were generated from polyA-tailed long non-coding RNAs (lncRNAs) (Additional file 11). The accumulation patterns of these polyA-tailed lncRNAs were similar to those of the phasiRNAs with a later initiation of expression in O. glaberrima than in $O$. barthii, regarding the morphological stages (Fig. 3; Additional file 12). Furthermore, we investigated the accumulation levels of mRNAs corresponding to gene 


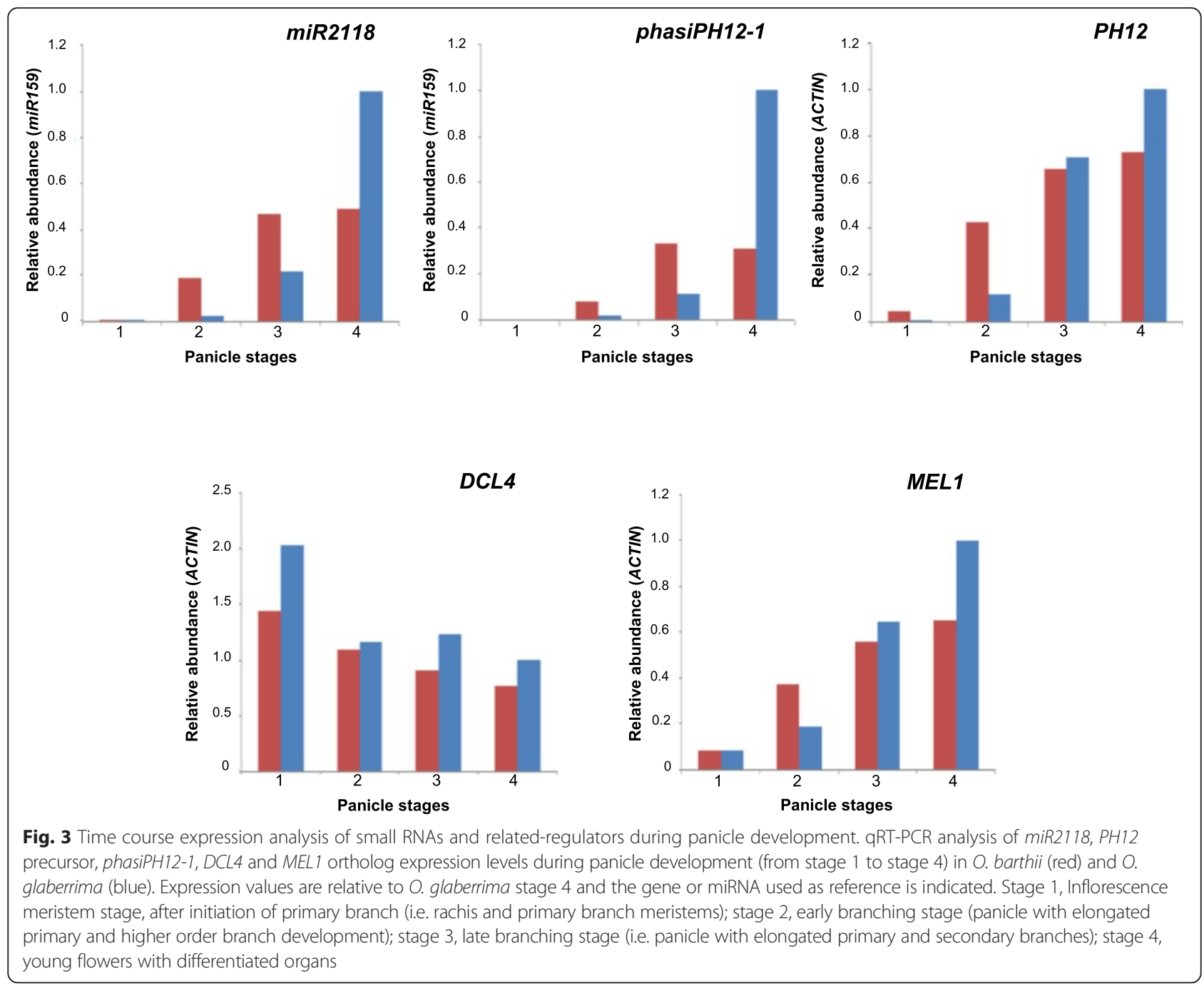

orthologs encoding other factors involved in the biogenesis of the miR2118-triggered 21-nt phased RNAs, such as the OsDCL4 DICER-like protein, and the gamete-specific Argonaute protein MEL1 (Song et al. 2012a, b; Komiya et al. 2014). The accumulation level of African rice OsDCL4 ortholog mRNAs decreases slightly over the 4 stages, while African rice $M E L 1$ ortholog mRNA accumulation increases from stage 1 to stage 4 in a similar pattern to the small RNAs and lncRNAs (Fig. 3; Additional file 12). Taken together, these data suggest that the accumulation levels of phasiRNAs may depend on miR2118, phased loci-associated lncRNAs and African rice MEL1 ortholog transcript accumulation levels rather than to those of the OsDCL4 ortholog.

To determine the spatial expression patterns of miR2118, phasiPH12-1 and PH12 lncRNAs during panicle development, in situ hybridization analysis was performed at different developmental stages of young panicles from the two species (Fig. 4). Similar patterns were observed for the two species with variations in spatial patterning of these factors. This analysis revealed that the onset of the miR2118-triggered 21-nt phasiRNA pathway is sequential in terms of initiation of expression. PH12 lncRNAs are detected first in spikelet meristems and are maintained in developing florets, but only in stamens and more specifically in the pollen sac. Subsequently, miR2118 specifically accumulates in the hypodermis of developing stamens (prior to pollen sac differentiation) at the early floret differentiation stage, then in the epidermis and the pollen sac in differentiated stamens at later stages. Finally, the phasiPH12-1 phasiRNAs are specifically located in pollen sac of differentiated stamens subsequent to miR2118 detection (Fig. 4). Eventually, all three signals were observed at the same location at later stages (i.e. pollen sac).

Our spatial expression analysis of miR2118, phasiPH12-1 and PH12 IncRNA and their differential 
(A)

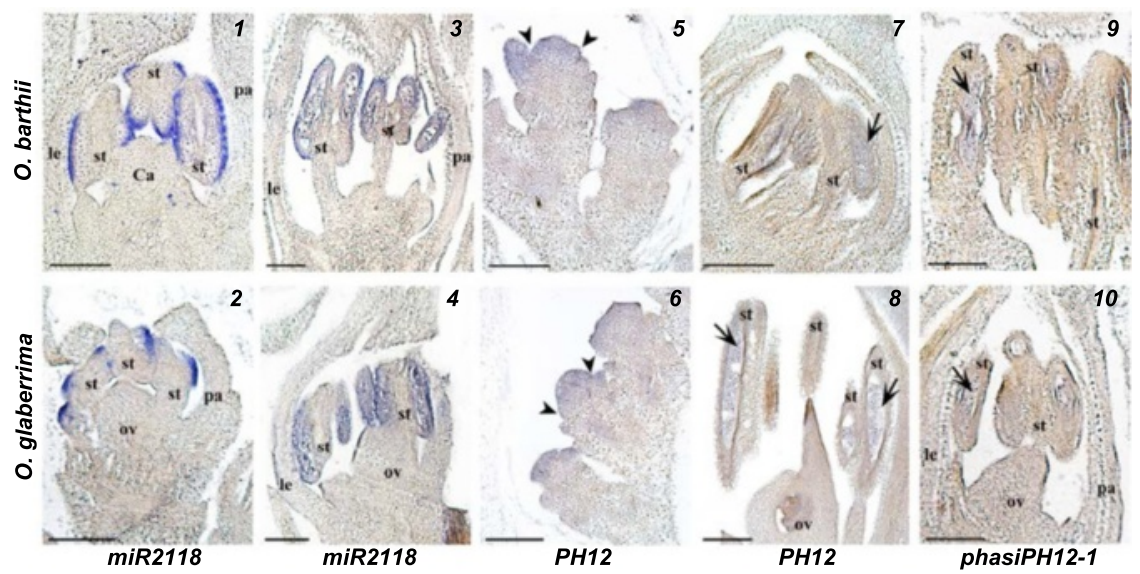

(B)

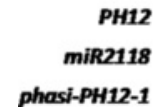

Fig. 4 In planta expression analysis of small RNAs and related-regulators during panicle development. a In situ hybridization of mature miR2118 (1-4), PH12 mRNA precursor (5-8) and phasiPH12-1 (9-10) in spikelet meristem (SMs), floret meristem (FMs) and differentiated floret in O. barthii (B88) and O. glaberrima (CG14). Mature miR2118 transcripts were detected in the outer cell layer of stamens (i.e. hypodermis) (1-2) and later in pollen sac (3-4). Precursor PH12 mRNAs were detected in the spikelet meristem (5-6) and in pollen sac of the anthers (arrow) (7-8). The phasiPH12-1 small RNAs were detected in the pollen sac of the anthers in florets (9-10). This panel of photos illustrates stages for which the transcripts were detected. le, lemma; pa, palea; st, stamen; ca, carpel; ov, ovule. Arrowheads, lemma and palea primordium formation in SMs; arrow, anthers. Scale bars: 100 m. b Schematic view of the expression profiling of PH12 mRNA precursor, mature miR2118 and phasiPH12-1 regarding meristem state and developmental timing based on in situ hybridization data. Blue boxes illustrate transcript detection periods. BM: branch meristem; SM: spikelet meristem; FM:

floret meristem

accumulation patterns with respect to the morphological stages of panicle development between the two species indicates that at the branching stage, the gameteassociated small RNAs are initiated later and expressed at lower levels in O. glaberrima than in O. barthii. This suggests that for the same morphological stage of panicle development, stamen differentiation, and therefore floret differentiation, might be delayed in O. glaberrima with respect to its wild relative, $O$. barthii.

\section{Variations in expression of a spikelet-related gene during panicle development}

To investigate this hypothesis further, we analysed the expression pattern of orthologs of the $O$. sativa Efunction MADS-box LEAFY HULL STERILE1 (LHS1)/ OsMADS1 gene that promotes the determination of meristems (i.e. spikelet and floret meristems) (Jeon et al. 2000; Cui et al. 2010; Khanday et al. 2013). Using in situ hybridization, we investigated the expression pattern of the LHS1 ortholog in O. barthii and O. glaberrima panicles at the early branching stage. In the two species,
LHS1 mRNA was detected specifically in SMs (Fig. 5). However, while the LHS1 mRNA signal was observed in some lateral meristems in O. glaberrima, it was detected in all lateral meristems in O. barthii (Fig. 5). This supports the hypothesis that for the same morphological stage, all terminal and lateral meristems had acquired the spikelet fate in $O$. barthii, whereas in O. glaberrima few had done so. These observations are also in agreement with the gamete-associated small RNA profiling data obtained.

\section{Discussion}

Conservation of miR2118-triggered 21-nt phasiRNAs in Oryza species

Using panicle-derived small RNA transcriptome sequencing in O. glaberrima and its wild ancestor, O. barthii, we showed that $29 \%$ of the 21-nt small RNA population is drastically repressed (or non activated) in cultivated genotypes during the branching stage of panicle development. This large difference in expression pattern suggests a change in regulation of key regulatory molecules(s). The 


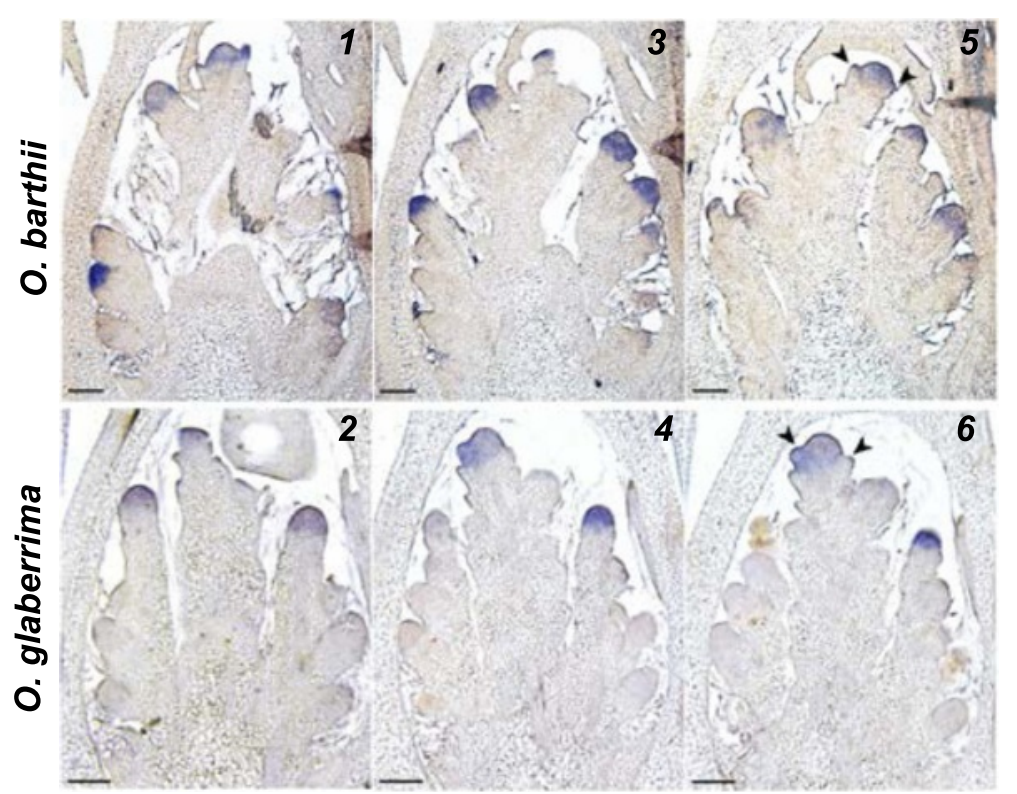

Fig. 5 In situ expression analysis of LHS1 gene in young panicles from African rice. In situ hybridization of LHS1 transcripts in serial sections of spikelet meristems (SMs) of O. barthii (1-3) and O. glaberrima (4-6); arrowheads, lemma and palea primordium formation in SMs. Scale bars: $100 \mu \mathrm{m}$

altered fraction of small RNAs corresponds mainly to 21nt phasiRNAs generated from nearly a thousand noncoding RNA loci, associated with altered expression of the 22-nt microRNA miR2118. This is in agreement with observations in $O$. sativa that panicle-specific 21 -nt phasiRNAs are produced from multiple non-coding RNA loci through an RDR6/DCL4-dependent pathway triggered by the microRNA miR2118 (Johnson et al. 2009; Song et al. 2012a, b). The synthesis of secondary siRNAs (or phasiRNAs) in reproductive organs from non-coding loci has only been reported in rice, maize and Brachypodium, suggesting a recent origin of these secondary siRNAs from a common ancestor of grasses (Zheng et al. 2015; Johnson et al. 2009; Song et al. 2012a, b; Zhai et al. 2015, Vogel et al. 2010). However, the low conservation of these loci across the group suggests that they may have speciesspecific functions (Zheng et al. 2015;, Komiya et al. 2014). Interestingly, miR2118-triggered secondary siRNA synthesis is also conserved across distantly related species, such as Medicago truncatula, tobacco and tomato. However, in these eudicot species, the miR2118 family members (including miR482 family members) were recruited to initiate secondary siRNAs on mRNAs encoding NBS-LRR proteins involved in disease resistance (Zhai et al. 2011; Li et al. 2012; Shivaprasad et al. 2012).

Our study revealed conserved features of panicleassociated 21-nt phasiRNAs and associated loci between Oryza species. However, this analysis was limited to the fraction of small RNAs corresponding to O. barthii and O. glaberrima phasiRNAs conserved in O. sativa (i.e. mapped to the O. sativa genome), and does not provide a complete overview of the complexity of phased loci in African rice species and their evolution during the African domestication process. The genome sequence of O. glaberrima accession CG14 was recently published (Wang et al. 2014), however, we obtained better mapping results from our small RNA sequence data using the reference genome O. sativa ssp japonica cv Nipponbare MSU v7.0 than the O. glaberrima CG14 released sequence: $72.1 \%$ of small RNA reads from both African species using Nipponbare MSU v7.0 sequence in contrast to $61.6 \%$ and $56.2 \%$ from O. barthii and O. glaberrima respectively using the $O$. glaberrima reference genome AGl1.1. For this reason, we continued to perform our small RNA sequence data analysis using the $O$. sativa Nipponbare reference genome. Nevertheless, it might be expected that higher diversification occurred between Asian and African rice since they diverged about 1 million years ago, whereas $O$. glaberrima and $O$. barthii diverged about 3000 years ago. Consequently, our quantitative analysis in African rice will be only marginally biased by evolutionary differences at the sequence level between the two African rice species. However, the number of overlapping phased loci between African rice species and $O$. sativa is quite low. About 1136 phased loci were identified from 4-cm long panicles of $O$. sativa ssp indica var 93-11. However, only 416 out of the 892 detected phased loci in African species overlapped phased loci detected in O. sativa spp indica var 93-11 (Song et al. 2012a). This may be 
because they were identified in a different way, i.e. based on different stages of panicle development. It should also be noted that more small RNA sequences were characterized in our study. Even if sequences are conserved between Oryza species, it cannot be ruled out that species-specific expression patterns of these lncRNA loci may occur. Moreover, $40 \%$ of the detected loci associated with differentially expressed 21-nt phasiRNAs between the two African species had no miR2118 recognition site motif, suggesting a potentially distinct mechanism of production. Another possible explanation would be that $m i R 2118$ recognition sites are present in the African rice genome at these loci but not in the $O$. sativa genome. In addition, in our comparative analysis, $48 \%$ of the over-expressed 21-nt small RNAs in $O$. barthii mapping to the $O$. sativa genome were not associated with the detected phased loci, and also originated mainly from unannotated regions of the genome. It is possible that these remaining over-expressed 21-nt small RNAs belong to phased loci specific to African rice genomes that were not detectable in our analysis because of sequence divergence from the O. sativa reference, or that they are associated with non-coding loci unrelated to phased small RNA generating loci. Recently, Zhang et al. (2014b) showed that a large set of long non-coding RNAs was expressed in anthers and pistils of $O$. sativa rice. Interestingly, only 122 of the 1624 reported lncRNA loci were associated with small RNAs (Zhang et al. 2014b). In agreement with this, only a few of the reported lncRNA loci overlapped with the miR2118-associated phasiRNAgenerating loci in O. sativa and African rice species (data not shown). This suggests that these lncRNAs are mostly not associated with the production of phasiRNAs or other small RNAs and may act through other mechanisms during plant development.

\section{The miR2118-triggered 21-nt phasiRNAs are markers of male-gametogenesis in African rice}

The function of the 21-nt phasiRNAs during panicle development is still unclear. MicroRNA miR2118 has been reported to be preferentially expressed in rice and maize stamens, suggesting a role in male gametogenesis (Song et al. 2012a). Recently, it was shown that the function of this class of phased small RNA was dependent on the germ line-specific Argonaute (AGO) protein MEL1 through a direct interaction between MEL1 and phasiRNAs (Komiya et al. 2014). Moreover, as mature miR2118 was detected in the MEL1-binding small RNA fraction, it was argued that MEL1 might play a role in the first steps of the 21-nt phasiRNA biogenesis pathway as the AGO protein in the miR2118-driven RISC triggering to the first cleavage of lncRNA precursors (Komiya et al. 2014). This is in agreement with the expression pattern observed for MEL1 and miR2118. MEL1 mRNAs were observed to be first detectable by in situ hybridization in the hypodermis of developing stamen primordia in a spotty pattern, similarly to that which we observed for miR2118 microRNA, later becoming restricted to microsporangia and pollen sac (Nonomura et al. 2007). In our study, while PH12 precursor lncRNAs were detected in the spikelet meristem differentiation stage, phasiPH12-1 was only detected in the stamens of differentiating florets and co-localized with $\mathrm{PH} 12$ precursor lncRNAs, but only after $m i R 2118$ was observed to accumulate. The latter was first limited to the hypodermis of differentiating stamens and extended to the pollen sac in later stages. Together, these data suggest that the 21-nt phasiRNA regulatory network is initiated early during panicle development from the stage of spikelet meristem establishment, before miR2118 and MEL1 expression, through the induction of lncRNA precursor expression. The coexpression of $m i R 2118$ and MEL1 in the hypodermis of the differentiating stamen would result in the initiation of the 21-nt phasiRNA biogenesis pathway, leading to the accumulation of the phasiRNAs. However, the transacting factors involved in the regulation of the lncRNA precursors are still unknown. Zhai et al. (2015) have recently shown that maize miR2118-triggered 21-nt phasiRNAs correspond to premeiotic siRNAs in anthers, with a similar spatial patterning observed in rice, indicating a conservation of the spatial regulation of this pathway in grasses.

Although direct evidence is still lacking for the involvement in male gametogenesis of miR2118 and the associated phasiRNAs and lncRNA precursors, their involvement is supported by the phenotypes of $r d r 6$ and dcl4 mutants affected in flower and stamen development (Liu et al. 2007; Song et al. 2012b). Moreover the mel1 mutant phenotype indicates that the MEL1 AGO protein mediates the regulation of germ-line mother cell development and meiosis in both male and female organs, suggesting that the MEL1-phasiRNA complexes may be involved in these processes (Nonomura et al. 2007; Komiya et al. 2014). The remaining question is whether these phasiRNAs can be considered as ta-siRNA (secondary siRNAs targeting other mRNAs). The predominant accumulation of MEL1 protein in the cytoplasm would favour a trans-acting function of MEL1-phasiRNA complex on mRNAs but without cleavage activity of the putative targets (Komiya et al. 2014; Zhai et al. 2015).

\section{Differential rate of determinate fate acquisition in the panicle meristems of African rice}

Our comparative analysis of small RNA expression with respect to the development of the African rice panicle suggests that the spikelet/floret fate acquisition rate differs between the two species: for a similar morphological 
complexity during the early branching stage, all meristems are converted into spikelets in $O$. barthii whereas only those of the apical part of the panicle branches are converted in O. glaberrima, as suggested by the expression pattern of the spikelet-associated MADS-box gene LHS1/OsMADS1. This difference was paralleled by the later initiation of expression and lower accumulation during the branching stage of the 21-nt phasiRNA pathway members (miR2118, MEL1, IncRNAs and phasiR$N A s$ ) in O. glaberrima, as well as the lncRNA precursors expressed in spikelet meristems. The differential rate of spikelet transition observed between the two species may be explained by a shorter duration of development in O. barthii than in O. glaberrima but also possibly by the synchronous transition of the apical and lateral meristems from the indeterminate to the determinate state in O. barthii.

Theoretical modelling of inflorescence architecture suggests that inflorescence branching complexity and its evolution depends on differences in the timing of floral (i.e. determinate) fate acquisition in apical and/or lateral meristems (Prusinkiewicz et al. 2007). This biological basis is supported by the analysis of various mutants affected in floral meristem identity in different species, notably in Arabidopsis thaliana, Antirrhinum majus, petunia and tomato (Koes 2008; Moyroud et al. 2010; Park et al. 2014). A comparative study of the diversity of inflorescence architecture in tomato based on meristemspecific transcriptome analysis provided support for this model (Park et al. 2012). In the case of the grass inflorescence and more specifically the rice panicle, it was also reported that variations in panicle architecture depended on the activity or expression levels of both meristem fate controlling genes and branch-promoting genes (Kyozuka et al. 2014; Zhang and Yuan 2014). In the context of our comparative analysis of African rice species, a differential rate of determinate fate acquisition in panicle meristems would result in a longer or higher rate of branching activity in O. glaberrima compared to O. barthii, leading to a higher branch complexity in the domesticated species. However, further functional studies are required to define the link between the major regulatory changes of spikeletrelated gene expression and the panicle phenotypic variations associated with domestication.

\section{Conclusions}

We provide evidence that the male-gametogenesisspecific 21 -nt phasiRNA pathway triggered by miR2118 is conserved in both wild relative and domesticated African rice species. Our study shows that the onset of the miR2118-triggered 21-nt phasiRNA pathway is sequential in terms of initiation of expression with variations in spatial patterning of these factors. This pathway is initiated when spikelet meristems are established, through the activation of lncRNA precursor expression. Our study provides evidence that the differential expression of the miR2118-triggered 21-nt phasiRNA pathway in $O$. barthii and O. glaberrima may be associated with different rates of determinate meristem fate acquisition during branching stage of panicle development. It will be of great interest to determine whether a similar scenario has occurred in the Asian rice species (O. sativa vs. O. rufipogon), so as to determine whether or not the phenotypic convergence of panicle development observed between the two domestication processes is paralleled by similar changes in the expression of molecular regulatory components associated with spikelet differentiation and panicle architecture complexity.

\section{Methods}

Plant materials and panicle sampling

For Illumina sequencing, 10 accessions of $O$. glaberrima and 10 accessions of $O$. barthii (see Additional file 1: Table S1) were grown in the greenhouse at IRD, Montpellier. Around 15 panicles from each accession were collected from 4 to 15 days after induction, to obtain homogeneous representation of early developmental stages corresponding to early rachis elongation to early floret differentiation.

For histological analysis and expression analysis, CG14 and B88 plants were grown in growth chamber at IRD, Montpellier (France) with a 14-10 h day/night cycle at $32{ }^{\circ} \mathrm{C} / 28{ }^{\circ} \mathrm{C}$ and humidity at $60 \%$. Flowering was induced by short day conditions (10-14 h day/night cycle). Panicles were collected at 4 different morphological stages: stage 1 , inflorescence meristem stage, after initiation of primary branch (i.e. rachis and primary branch meristems); stage 2 , early branching stage (i.e. panicle with elongated primary and higher order branch development); stage 3, late branching stage (i.e. panicle with elongated primary and secondary branches); stage 4, young flowers with differentiated organs (Additional file 10).

\section{Illumina sequencing and data processing}

Total RNAs (including small RNAs) were extracted using an RNeasy Plant Mini Kit with RLT and RWT buffers (Qiagen, France). DNase treatments were performed using the RNAeasy-free DNase set (Qiagen, France). Two bulks of total RNAs corresponding to a mix of total RNAs from the 10 accessions of the two species were used for sequencing. Purified small RNA sequencing was performed by Eurofins/MWG Operon (Germany) on an Illumina Hi-seq 2000 using the Tru$\mathrm{Seq}^{\text {Tm }}$ SBS v5 sequencing kit. The complete raw dataset is available in NCBI Gene Expression Omnibus repository [GEO: GSE48346]. The raw data were trimmed by removing adapter sequences and low quality sequences 
using CutAdapt (Martin 2011). All the trimmed reads ranging from 18 to 28 nucleotides were clustered and mapped to $O$. sativa ssp japonica cv Nipponbare genome (MSU release version 7; http://rice.plantbiology.msu.edu/) using BLAST (Altschul et al. 1990) (Additional file 4) in order to evaluate library quality. The 18-28 nucleotide reads were them annotated through a filtering process using successive hierarchical BLAST (Additional file 4) versus (in order) miRBase v17.0 (Kozomara and Griffiths-Jones 2011), Rfam v7, a home-made repeat database (successive curated concatenation of RetrOryza, RepBase, TREP and TIGRRepeats), CDS then gene features (i.e. introns and UTRs) from Oryza sativa ssp japonica var Nipponbare MSU v7.0 annotation, and finally the MSU v7.0 rice genome for the sequences not similar to miRNAs, non coding RNAs, Repeat-associated sequences and genes. BLAST filtering was done using Oryza genus data form the different databases (i.e. mainly $O$. sativa). Mapping of the sequences to the $O$. sativa MSU v7.0 reference genome was done for the last three steps of our annotation pipeline. This genome was used as a reference because $(i)$ of its equal divergence to the two species we analyzed (avoiding favoring one species in mapping analyses rather than the other one), and (ii) because of the larger resources in terms of annotation, expression data and genomic information available for this species. The BLAST and post-filter parameters used were probability of $85 \%$, e-value of $10^{-3}$, on a size of $85 \%$ of the reads (minimum size of 16). The same $B L A S T$ parameters were used throughout the analysis. Mapping from O. glaberrima and O. barthii small RNA sequences was then compared and filtered using a series of homemade Perl scripts (available on demand). Comprehensive analysis of the miRNA-associated small RNAs from African rice species was limited to the families present in O. sativa, and did not include diverse isoforms. For the same reason, potential African rice specific miRNAs were not investigated. The 21-mers were used in phasing analysis with the ta-si Prediction tool from the UEA $s R N A$ workbench facilities (http:// srna-workbench.cmp.uea.ac.uk/; Stocks et al. 2012). A post-analysis manual filtering was performed for sequences mapping to more than 10 loci in genome, as well as telomere-associated loci, leading to the deletion of 123 loci from the 1015 initial loci reported in $t a-s i$ Prediction tool outputs. Once the loci were identified, we used the EMBOSS software suite v6.5.7.0 (Rice et al. 2000) to extract $-500 /+500$ bases around each locus, and treated them using MEME v4.8.1 (Bailey and Elkan 1994). Then, the loci sequences were used as a BLAST database to add other 21-nt sequences that were not identified originally as phased (in order to by-pass the genome divergence), in two pass: first we select only new sequences with $100 \%$ of identity on $85 \%$ of their length (minimum HSP length of 16), then we change the threshold to $85 \%$ of identity on $85 \%$ of their length (min HSP length of 16). Finally, statistical tests of all the processed data were performed using $g$-test and a fixed $p$-value of $10^{-3}$. Depending on the experiment, the degree of freedom was adjusted but was generally 1 . All the calculations were performed using homemade Perl scripts and CPAN statistical modules.

\section{Quantitative RT-PCR analysis}

Total RNAs (including small RNAs) were extracted as described before, from the 4 individual morphological stages of young panicles of $O$. glaberrima (CG14) and $O$. barthii (B88). First-stand cDNA was synthesised using SuperScript III cDNA First-strand synthesis system (Invitrogen). Quantitative stem-loop RT-PCR analyses on small RNAs were performed using $100 \mathrm{ng}$ of total RNA according to Varkonyi-Gasic et al. (2007) with reverse transcription at $42{ }^{\circ} \mathrm{C}$ in conjunction with small RNA-specific stem-loop primers. Quantitative RT-PCR analyses on mRNAs were performed using $1 \mu \mathrm{g}$ of total RNA in conjunction with polydT or random hexamer primers according to the manufacturer's instructions. qRT-PCRs were performed using LightCycler 480 thermocycler (Roche, France) in conjunction with SYBR Green I master mix (Roche, France) in $8 \mu \mathrm{L}$ reaction mix containing $2 \mu \mathrm{L}$ of diluted RTs and $0.8 \mu \mathrm{L}$ of forward and reverse primers at $10 \mu \mathrm{M}$. The qPCR amplification conditions include 3 stages: pre-incubation $\left(95^{\circ} \mathrm{C}\right.$ in $10 \mathrm{~min})$; amplification with 45 cycles $\left(95^{\circ} \mathrm{C} 15 \mathrm{~s}\right.$ and $\left.60{ }^{\circ} \mathrm{C} \mathrm{30s}\right)$; melting curve $\left(95^{\circ} \mathrm{C} 5 \mathrm{~s}\right.$ and $\left.70{ }^{\circ} \mathrm{C} 1 \mathrm{~min}\right)$. In stem-loop qRT-PCR, the levels of miRNA were normalized by mature miR159 expression level. In classic qRTPCR, mRNAs were normalized to the rice Actin gene (LOC_Os03g50885.1). Each set of experiments was repeated three times, and the relative quantification method with efficiency corrected calculation model (Souaze et al. 1996) was used to evaluate quantitative variations. The primers used are listed in Additional file 1: Table S5.

\section{In situ hybridizations}

PCR amplifications were performed with gene-specific antisense primers tailed with a T7 RNA polymerase binding site (see Additional file 1: Table S5 for primer sequences). The resulting DNA fragments were used directly as templates for synthesizing antisense ribo-probes incorporating UTP-digoxigenin (Roche) as the label in conjunction with a T7 Maxi Script kit (Ambion). For miR2118, PH12 precursor and phasiPH12-1 detection, $0.02 \mu \mathrm{M}$ of a $5^{\prime}$ digoxigenin-labeled LNA probe complementary to the target (see Additional file 1: Table S3 for primer sequences) was used. In situ hybridization experiments were carried out as described by Adam et al. 
(2011). Detection was performed using the Vector Blue Alkaline Phosphatase Substrate Kit III (Vector Laboratories). Slides were observed and photographed by Evolution MP5.0 colour Media Cybernetics camera in conjunction with a Leica DMRB microscope and images were processed using Photoshop CS6.

\section{Additional files}

Additional file 1: Table S1. List of genotypes used in this study. Table S2. Summary statistics of small RNA libraries. Table S3. Summary of sequence number (clusters and reads) mapped to O. sativa Nipponbare reference genome (MSU7.0) and O. glaberrima CG14 reference genome for $O$. barthii and $O$. glaberrima small RNA sequences. Table S4. Summary of sequence number (clusters and reads) in each annotation class (i.e. sequence databases) for $O$. barthii and $O$. glaberrima small RNA sequences based on BLAST-filtering. Table S5. List of primers used in this study. The bases in brackets represent the ones that were modified LNAs. The sequences in italic correspond to stem-loop region for stem-loop RT-PCRs. The underline sequences correspond to T7 RNA polymerase binding site used for RNA probe synthesis. (PDF $191 \mathrm{~kb}$ )

Additional file 2: Size distribution, mapping and annotation of $O$. barthii and O. glaberrima panicle-derived small RNAs on O. sativa Nipponbare genome. (a) Small RNA size distribution of clusters (distinct) and reads (abundance). The size of small RNAs was plotted versus frequency (percentage relative to total abundance). (b) Mapping rate (percentage relative to total number) of clusters (i.e. distinct sequences) and total reads on O. sativa nipponbare MSU7.0. The percentage values are indicated in the bars. (c) Small RNA annotation frequency (percentage relative to total abundance) of clusters (distinct) and individual reads (abundance). The percentage values are indicated in the bars. (d) Size distribution of the annotated small RNA clusters (i.e. distinct sequences) according the annotation classes. (e) Size distribution of the annotated small RNA sequences (i.e. abundance/reads) according the annotation classes. (PDF $333 \mathrm{~kb}$ )

Additional file 3: Genomic distribution and abundance of $O$. barthii and O. glaberrima panicle-derived small RNAs on O. sativa nipponbare genome. (a) Distribution and abundance of $O$. barthii reads vs. O. sativa nipponbare genome MSU7.0. (b) Distribution and abundance of 0 . glaberrima reads vs. O. sativa nipponbare genome MSU7.0. (PDF 1091 kb)

Additional file 4: Bio-informatic workflow for small RNA analysis. (PDF $158 \mathrm{~kb}$ )

Additional file 5: Relative abundance of small RNAs between O. barthii and O. glaberrima. (a) Relative abundance of small RNAs between $O$. barthii $(\mathrm{Ob})$ and $\mathrm{O}$. glaberrima $(\mathrm{Og})$ according to their size (from 18- to 28-nt small RNAs). LogPlots of normalized abundance of distinct small RNA sequences. (b) Relative abundance of the annotation classes of 21-nt small RNAs between 0 . barthii $(\mathrm{Ob})$ and O. glaberrima $(\mathrm{Og})$. LogPlot of normalized abundance of distinct small RNA sequences. Black dots represent global 21-nt small RNAs, and red dots the class of annotated 21-nt small RNAs. (c) Relative abundance of the annotation classes of 24-nt small RNAs between 0 . barthii $(\mathrm{Ob})$ and O. glaberrima $(\mathrm{Og})$. LogPlot of normalized abundance of distinct small RNA sequences. Black dots represent whole 24-nt small RNAs, red dots represent the class of annotated 24-nt small RNAs. (PDF $1571 \mathrm{~kb}$ )

Additional file 6: 21-nt small RNAs associated to phased loci and mature miRNA families detected in young panicles of 0 . glaberrima and O. barthii. (a) O. sativa Nipponbare loci associated with 21-nt phased and unphased siRNAs from $O$. barthii and $O$. glaberrima detected using sRNAworkbench facilities. (b) Normalized count of reads (relative abundance for 2 millions of total reads) associated with mature miRNA families in relation to small RNA size. (PDF 383 kb)

Additional file 7: Genomic distribution, abundance and complexity of O. barthii and O. glaberrima 21-nt phased small RNAs on O. sativa nipponbare genome (MSU 7.0). (a) Genomic distribution and abundance of $O$. barthii and $O$. glaberrima 21 -nt phasiRNAs on $O$. sativa nipponbare genome (MSU 7.0). (b) Distribution of number of detected 21-nt phasiRNAs from African species per phased locus on $O$. sativa nipponbare genome (MSU 7.0). (PDF 547 kb)

Additional file 8: Sequences and phasiRNA abundances of the 4 phased loci used for validation. The 4 phased loci reported here were used for stem-loop RT-PCR (phasiRNAs) and classical RT-PCR (IncRNAs) validation. The positions of the phased loci on MSU7.0 chromosomes are indicated. The alignment between miR2118f and the phased locus is indicated. The miR2118 recognition site is in blue. The phasiRNAs detected in the plus strand are highlighted alternately in green and in red. The phasiRNAs detected in the minus strand are underlined alternately in green and in red. The phasiRNA boxed in yellow is the most abundant one and the one used for stem-loop RT-PCR validation and qRT-PCR analysis. Histograms illustrate the relative abundance of the detected phasiRNAs per locus in the two species (PDF $159 \mathrm{~kb}$ )

Additional file 9: Features of $O$. barthii and $O$. glaberrima panicle-derived microRNAs and abundance of sequences related to microRNA precursor sequences. (a) Size distribution of clusters (distinct) and reads (abundance) related to $O$. sativa microRNAs in $O$. barthii and O. glaberrima. (b) Relative abundance of $O$. barthii and $O$. glaberrima reads related to $O$. sativa microRNA precursors. The precursors are classified according to the abundance distribution pattern as defined by Jeong et al. (2011) (i.e. canonical, variant, siRNA-like). (PDF $200 \mathrm{~kb}$ )

Additional file 10: Histological description of selected developmental stages of African rice panicles. O. barthii: 1,3,5,7,9,11; O. glaberrima: $2,4,6,8,10,12$; stage 1 : unbranched stage with elongation of rachis meristem (arrowheads) and formation of primary branch meristems $\left(^{*}\right)$ $(1,2) ;$ stage 2: early branching stage with rachis meristem (arrowheads) and elongating primary branches $(3,4)$. At the end of this stage, secondary branches (white ${ }^{*}$ ) are initiated from PBs $\left(^{*}\right)(5,6)$; stage 3 : late branching stage with elongated secondary branch and spikelet meristem (SM) and floret meristem (FM) differentiation (7,8); stage 4: floret organ differentiation/ development $(9,10)$; mature stage: 11 and 12 . White arrowhead: vestige of aborted rachis meristem. Scale bar: $100 \mu \mathrm{m}$. (PDF 1544 kb)

Additional file 11: RNA-seq data validation by northern-blotting and semi-quantitative RT-PCR for miRNAs, phasiRNAs and ncRNAs associated with phased loci in panicle-derived small RNA bulks from 0 . barthii $(O b)$ and O. glaberrima $(\mathrm{Og})$. (a) Northern blot hybridization using miR2118 as probe. U6 probe was used as control. (b) Stem-loop RT- PCR analysis of various miRNAs and phasiRNAs. miR159a probe was used as a loading control. (c) Classic RT-PCR analysis of ncRNAs associated with 21-nt phased small RNA loci using from polydT primer (dT) or random hexamer primers (RH) for the RTs. The ACTIN gene (Os03g50885) was used as a loading control. (d) Test of specificity of stem-loop RT-PCRs against phasiRNAs on PH12 and PH779 loci, in conjunction with phasiRNA specific forward primer (PH12-F and PH779-F primers; phasiRNA label) and long ncRNA forward primer (PH12-F2 and PH779-F2 primers; long ncRNA label). Controls: 1. RT reaction using O. glaberrima RNA bulk without stem-loop RT primer; 2. RT reaction using $O$. barthii RNA bulk without stem-loop RT primer; 3 . RTPCR without stem-loop RT matrix; $\varnothing$ : RT-PCR without RT. See Additional file 1: Table S5 for primer and probe sequences. (PDF $1888 \mathrm{~kb}$ )

Additional file 12: Expression patterns of phasiRNAs and IncRNA precursors associated with 21-nucleotide phased loci in O. glaberrima and O. barthii. qRT-PCR analysis of 21-nucletiode phasiRNAs expression levels (a) and IncRNAs (b) from 3 distinct phased loci (PH557, PH612 and PH779) during panicle development in 0 . barthii (red) and O. glaberrima (blue). Expression values are relative to 0 . glaberrima stage 4 and the ACTIN gene or miR159 miRNA used as reference is indicated. Panicle stages: Stage 1, Inflorescence meristem stage, after initiation of primary branch (i.e. rachis and primary branch meristems); stage 2, early branching stage (panicle with elongated primary and higher order branch development); stage 3, late branching stage (i.e. panicle with elongated primary and secondary branches with spikelets and beginning of floret differentiation); stage 4, young flowers with differentiated organs. See Additional file 1: Table S5 for primer sequence. (PDF 56 kb) 


\section{Authors' contributions}

SF established and conducted the bio-informatic pipeline analysis of the small RNA dataset; JS, VY and SF carried out the analysis of bio-informatic out-puts; TKN performed molecular analysis (PCR, RT-PCR, in situ hybridization); MS conducted the initial bio-informatic analysis of data set; $\mathrm{AH}$ performed the sampling and RNA preparation; JS and $\mathrm{VY}$ initiated and organized the project; GP, DNV and GA managed the project. JS, SF, TKN, VY, $\mathrm{AH}$ and GP wrote manuscript together. All authors approved the final manuscript.

\section{Acknowledgments}

We thank members of the lab for their help with plant care and RNA extractions. We thank members of IRD-Montpellier bioinformatics platform for their technical support. We also thank colleagues from IRD-Montpellier for critical reading of the manuscript. This project was jointly supported by Agropolis Fondation (through the «Investissements d'avenir » programme (ANR-10-LABX-0001-01)) and Fondazione Cariplo under the reference ID EVOREPRICE 1201-004. This work was also supported by the Institut de Recherche pour le Développement (IRD) institutional funding, Agropolis foundation (ARCAD project for post-doctoral fellowship of SDM), Vietnamese government (program 322 to TKN) and the Global Rice Science Partnership (GRiSP) scholarship program (TKN)

\section{Author details}

'IRD, UMR DIADE, 911, avenue Agropolis, BP64501, F-34394 Montpellier, Cedex 5, France. ${ }^{2}$ LMI RICE, National Key Laboratory for Plant Cell Biotechnology, Agronomical Genetics Institute, Pham Van Dong road, Hanoi, Vietnam. ${ }^{3}$ Université de Montpellier, UMR DIADE, Place Eugène Bataillon, F-34095 Montpellier, Cedex 5, France. ${ }^{4}$ Present address: INRA, Université de Lorraine, UMR 1136 Interactions Arbres/Microorganismes, F-54280 Champenoux, France.

Received: 19 December 2015 Accepted: 6 March 2016 Published online: 12 March 2016

\section{References}

Adam H, Marguerettaz M, Qadri R, Adroher B, Richaud F, Collin M, Thuillet AC, Vigouroux Y, Laufs P, Tregear JW, Jouannic S (2011) Divergent expression patterns of miR164 and CUP-SHAPED COTYLEDON genes in palms and other monocots: implication for the evolution of meristem function in angiosperms. Mol Biol Evol 28:1439-1454

Allen E, Xie Z, Gustafson AM, Carrington JC (2005) MicroRNA-directed phasing during trans-acting siRNA biogenesis in plants. Cell 121:207-221

Altschul SF, Gish W, Miller W, Myers EW, Lipman DJ (1990) Basic local alignment search tool. J Mol Biol 215:403-410

Arikit S, Zhai J, Meyers BC (2013) Biogenesis and function of rice small RNAs from non-coding RNA precursors. Curr Opin Plant Biol 16:170-179

Bailey TL, Elkan C (1994) Fitting a mixture model by expectation maximization to discover motifs in biopolymers. In: Altman R, Brutlag D, Karp P, Lathrop R, Searls D (eds) Proceedings of the Second International Conference on Intelligent Systems for Molecular Biology. AAAI Press, Menlo Park

Caicedo AL, Williamson SH, Hernandez RD, Boyko A, Fledel-Alon A, York TL, Polato NR, Olsen KM, Nielsen R, McCouch SR, Bustamante CD, Purugganan MD (2007) Genome-wide patterns of nucleotide polymorphism in domesticated rice. PLoS Genet 3:1745-1756

Carroll S (2008) Evo-Devo and an expanding evolutionary synthesis: a genetic theory of morphological evolution. Cell 134:25-36

Cui R, Han J, Zhao S, Su K, Wu F, Du X, Xu Q, Chong K, Theissen G, Meng Z (2010) Functional conservation and diversification of class $E$ floral homeotic genes in rice (Oryza sativa). Plant J 61:767-781

Doebley J, Lukens L (1998) Transcriptional regulators and the evolution of plant form. Plant Cell 10:1075-1082

Huang X, Kurata N, Wei X, Wang Z-X, Wang A, Zhao Q, Zhao Y, Liu K, Lu H, Li W et al (2012) A map of rice genome variation reveals the origin of cultivated rice. Nature 490:497-501

Jeon JS, Jang S, Lee S, Nam J, Kim C, Lee SH, Chung YY, Kim SR, Lee YH, Cho YG et al (2000) leafy hull sterile 1 is a homeotic mutation in a rice MADS box gene affecting rice flower development. Plant cell 12:871-884

Jeong D-H, Park S, Zhai J, Gurazada SGR, De Paoli E, Meyers BC, Green PJ (2011) Massive analysis of rice small RNAs: mechanistic implications of regulated
microRNAs and variants for differential target RNA cleavage. Plant cell 23: 4185-4207

Johnson C, Kasprzewska A, Tennessen K, Fernandes J, Nan G-L, Walbot V, Sundaresan V, Vance V, Bowman LH (2009) Clusters and superclusters of phased small RNAs in the developing inflorescence of rice. Genome res 19 : $1429-1440$

Jones-Rhoades MW, Bartel DP, Bartel B (2006) MicroRNAS and their regulatory roles in plants. Ann Rev Plant Biol 57:19-53

Khanday I, Yadav SR, Vijayraghavan U (2013) Rice LHS1/OsMADS1 controls floret meristem specification by coordinated regulation of transcription factors and hormone signaling pathways. Plant Physiol 161:1970-1983

Koes R (2008) Evolution and development of virtual inflorescences. Trends Plant Sci 13:1-3

Komiya R, Ohyanagi H, Niihama M, Watanabe T, Nakano M, Kurata N, Nonomura K-I (2014) Rice germline-specific Argonaute MEL1 protein binds to phasiRNAs generated from more than 700 lincRNAs. Plant J 78:385-397

Kozomara A, Griffiths-Jones S (2011) miRBase: integrating microRNA annotation and deep-sequencing data. Nucl Acids Res 39:D152-D157

Kyozuka J, Tokunaga H, Yoshida A (2014) Control of grass inflorescence form by the fine-tuning of meristem phase change. Curr Opin Plant Biol 17: $110-115$

Li Y, Li C, Xia J, Jin Y (2011a) Domestication of transposable elements into MicroRNA genes in plants. PLOS ONE 6:e19212

Li Z-M, Zheng X-M, Ge S (2011b) Genetic diversity and domestication history of African rice (Oryza glaberrima) as inferred from multiple gene sequences. Theor Appl Genet 123:21-31

Li F, Pignatta D, Bendix C, Brunkard JO, Cohn MM, Tung J, Sun H, Kumar P, Baker B (2012) MicroRNA regulation of plant innate immune receptors. Proc Natl Acad Sci USA 109:1790-1795

Linares OF (2002) African rice (Oryza glaberrima): history and future potential. Proc Natl Acad Sci USA 99:16360-16365

Liu B, Chen Z, Song X, Liu C, Cui X, Zhao X, Fang J, Xu W, Zhang H, Wang X et al (2007) Oryza sativa dicer-like4 reveals a key role for small interfering RNA silencing in plant development. Plant Cell 19:2705-2718

Liu Y, Wang Y, Zhu QH, Fan L (2013) Identification of phasiRNAs in wild rice (Oryza rufipogon). Plant Signal Behav 8:e25079

Martin M (2011) Cutadapt removes adapter sequences from high-throughput sequencing reads. EMBnetwork J 18:10-12

Moyroud E, Kusters E, Monniaux M, Koes R, Parcy F (2010) LEAFY blossoms. Trends Plant Sci 15:346-352

Nabholz B, Sarah G, Sabot F, Ruiz M, Adam H, Nidelet S, Ghesquière A, Santoni S, David J, Glémin S (2014) Transcriptome population genomics reveals severe bottleneck and domestication cost in the African rice (Oryza glaberrima). Mol Ecol 23:2210-2227

Nogueira FTS, Madi S, Chitwood DH, Juarez MT, Timmermans MCP (2007) Two small regulatory RNAs establish opposing fates of a developmental axis. Genes Dev 21:750-755

Nonomura K-I, Morohoshi A, Nakano M, Eiguchi M, Miyao A, Hirochika H, Kurata N (2007) A germ cell specific gene of the ARGONAUTE family is essential for the progression of premeiotic mitosis and meiosis during sporogenesis in rice. Plant Cell 19:2583-2594

Orjuela J, Sabot F, Chéron S, Vigouroux Y, Adam H, Chrestin H, Sanni K, Lorieux M, Ghesquière A (2014) An extensive analysis of the African rice genetic diversity through a global genotyping. Theor Appl Genet 127:2211-2223

Park SJ, Jiang K, Schatz MC, Lippman ZB (2012) Rate of meristem maturation determines inflorescence architecture in tomato. Proc Natl Acad Sci USA 109: 639-644

Park SJ, Eshed Y, Lippman ZB (2014) Meristem maturation and inflorescence architecture - lessons from the Solanaceae. Curr Opin Plant Biol 17:70-77

Peng T, Lv Q, Zhang J, Li J, Du Y, Zhao Q (2011) Differential expression of the microRNAs in superior and inferior spikelets in rice (Oryza sativa). J Exp Bot 62:4943-4954

Prusinkiewicz P, Erasmus Y, Lane B, Harder LD, Coen E (2007) Evolution and development of inflorescence architectures. Science 316:1452-1456

Rice P, Longden I, Bleasby A (2000) EMBOSS: The European Molecular Biology Open Software Suite. Trends Genet 16:276-277

Second G (1982) Origin of the genic diversity of cultivated rice (Oryza spp.): study of the polymorphism scored at 40 isozyme loci. Jap J Genet 57:25-57

Shivaprasad PV, Chen H-M, Patel K, Bond DM, Santos BACM, Baulcombe DC (2012) A microRNA superfamily regulates nucleotide binding site-leucine-rich repeats and other mRNAs. Plant Cell 24:859-874 
Song X, Li P, Zhai J, Zhou M, Ma L, Liu B, Jeong D-H, Nakano M, Cao S, Liu C et al (2012a) Roles of DCL4 and DCL3b in rice phased small RNA biogenesis. Plant J 69:462-474

Song X, Wang D, Ma L, Chen Z, Li P, Cui X, Liu C, Cao S, Chu C, Tao Y et al (2012). Rice RNA-dependent RNA polymerase 6 acts in small RNA biogenesis and spikelet development. Plant J 71:378-389

Souaze F, Ntodou-Thome A, Tran CY, Rostene W, Forgez P (1996) Quantitative RT-PCR: limits and accuracy. Biotechniques 21:280-285

Stocks MB, Moxon S, Mapleson D, Woolfenden HC, Mohorianu I, Folkes L, Schwach F, Dalmay T, Moulton V (2012) The UEA sRNA workbench: a suite of tools for analyzing and visualizing next generation sequencing microRNA and small RNA datasets. Bioinformatics 28:2059-2061

Varkonyi-Gasic E, Wu R, Wood M, Walton EF, Hellens RP (2007) Protocol: a highly sensitive RT-PCR method for detection and quantification of microRNAs. Plant Meth 3:12

Vaughan DA, Lu BR, Tomooka N (2008) The evolving story of rice evolution. Plant Sci 174:394-408

Vogel JP, Garvin DF, Mockler TC, Schmutz J, Rokhsar D, Bevan MW, Barry K, Lucas S, Harmon-Smith M, Lail K et al (2010) Genome sequencing and analysis of the model grass Brachypodium distachyon. Nature 463:763-768

Wang Y, Shen D, Bo S, Chen H, Zheng J, Zhu Q-H, Cai D, Helliwell C, Fan L (2010) Sequence variation and selection of small RNAs in domesticated rice. BMC Evol Biol 10:119

Wang Y, Bai X, Yan C, Gui Y, Wei X, Zhu Q-H, Guo L, Fan L (2012) Genomic dissection of small RNAs in wild rice (Oryza rufipogon): lessons for rice domestication. New Phytol 196:914-925

Wang M, Yu Y, Haberer G, Marri PR, Fan C, Goicoechea JL, Zuccolo A, Song X, Kudrna D, Ammiraju JSS et al (2014) The genome sequence of African rice (Oryza glaberrima) and evidence for independent domestication. Nature Genet 46:982-988

Wei L, Yan L, Wang T (2011) Deep sequencing on genome-wide scale reveals the unique composition and expression patterns of microRNAs in developing pollen of Oryza sativa. Genome Biol 12:R53

Xia R, Meyers BC, Liu Z, Beers EP, Ye S, Liu Z (2013) MicroRNA superfamilies descended from miR390 and their roles in secondary small interfering RNA biogenesis in Eudicots. Plant Cell 25:1555-1572

Yan Y, Zhang Y, Yang K, Sun Z, Fu Y, Chen X et al (2011) Small RNAs from MITEderived stem-loop precursors regulate abscisic acid signaling and abiotic stress responses in rice. Plant J 65:820-828

Zhai J, Jeong D-H, De Paoli E, Park S, Rosen BD, Li Y, González AJ, Yan Z, Kitto SL, Grusak MA et al (2011) MicroRNAs as master regulators of the plant NB-LRR defense gene family via the production of phased, trans-acting siRNAs. Genes Dev 25:2540-2553

Zhai J, Zhang H, Arikit S, Huang K, Nan G-L, Walbot V, Meyers BC (2015) Spatiotemporally dynamic, cell-type-dependent premeiotic and meiotic phasiRNAs in maize anthers. Proc Natl Acad Sci USA 112:3146-315

Zhang D, Yuan Z (2014) Molecular control of grass inflorescence development. Ann Rev Plant Biol 65:553-578

Zhang Q-J, Zhu T, Xia E-H, Shi C, Liu Y-L, Zhang Y, Liu Y, Jiang W-K, Zhao Y-J, Mao S-Y et al (2014a) Rapid diversification of five Oryza AA genomes associated with rice adaptation. Proc Natl Acad Sci USA 111:E4954-E4962

Zhang Y-C, Liao J-Y, Li Z-Y, Yu Y, Zhang J-P, Li Q-F, Qu L-H, Shu W-S, Chen Y-Q (2014b) Genome-wide screening and functional analysis identify a large number of long noncoding RNAs involved in the sexual reproduction of rice. Genome Biol 15:512

Zheng Y, Wang Y, Wu J, Ding B, Fei Z (2015) A dynamic evolutionary and functional landscape of plant phased small interfering RNAs. BMC Biol 13:32

\section{Submit your manuscript to a SpringerOpen ${ }^{\mathcal{O}}$ journal and benefit from:}

- Convenient online submission

- Rigorous peer review

- Immediate publication on acceptance

- Open access: articles freely available online

- High visibility within the field

- Retaining the copyright to your article 